\title{
Miotomía peroral endoscópica (POEM) y piloromiotomía endoscópica peroral (G-POEM)
}

\author{
Óscar V. Hernández-Mondragón* y Luis F. García-Contreras \\ Departamento de Endoscopia Gastrointestinal, Hospital de Especialidades, Centro Médico Nacional Siglo XXI, Ciudad de México, Méx., México
}

\section{Resumen}

Desde la invención de la miotomía endoscópica a través de la boca (POEM) por Inoue en 2010, ha existido una evolución y expansión de su uso en distintas enfermedades gastrointestinales, pero siempre con un mismo principio: el túnel. En la semana de enfermedades digestivas (DDW) 2021, se presentaron 31 trabajos en relación con este tema. Revisaremos lo más relevante.

\section{Miotomía peroral endoscópica (POEM)}

Stavropoulos, et al. ${ }^{1}$ presentaron un estudio retrospectivo comparando enfermedad por reflujo gastroesofágico (ERGE) a corto y largo plazo en pacientes post-POEM; evaluaron las pHmetrías, endoscopias y cuestionarios clínicos de 704 procedimientos realizados en 11 años. Evaluaciones a los 4.7 meses y 56.7 meses en promedio después del POEM. La proporción de pHmetrías positivas fue menor en los estudios tardíos (47.6 vs. $33 \%$ ); $35 \%$ se normalizaron en la evaluación tardía; $71 \%$ presentaron menor puntaje en el cuestionario de síntomas y $57 \%$ tenían menor dosis de inhibidores de bomba de protones (IBP). Se sugiere que podría existir una potencial remodelación a nivel de la unión EG posterior al procedimiento y que podría generar una cicatriz que disminuiría el reflujo. Con estos hallazgos, se sugiere aplazar el estudio de pHmetría inicial 6 a 12 meses posteriores al POEM para no sobreestimar la incidencia de ERGE en estos pacientes.

Kolb, et al. ${ }^{2}$ realizaron un estudio de cohorte retrospectiva, para evaluar el tratamiento de la enfermedad por reflujo post-POEM y determinar los factores asociados para poder realizar un procedimiento antirreflujo. 132 POEM en 6 años, 87\% con acalasia, el resto con trastornos espásticos. El 13\% presentó ERGE refractario a IBP y se sometieron a funduplicatura transoral sin incisiones (TIF) en promedio 399 días posteriores al POEM; de estos solo $66 \%$ contaba con pHmetría con DeMeester positivo. De los pacientes sometidos a TIF 58\% tenia acalasia (tipo I, II y III) y $16 \%$ trastornos espásticos. El riesgo de presentar reflujo significativo que ameritara TIF fue mayor en trastornos espásticos y en acalasia tipo I. En el seguimiento a 6 y 12 meses post-TIF, solo 33\% lograron suspender/disminuir la dosis de IBP. Conclusión: Ios trastornos espásticos y acalasia tipo I sometidos a POEM presentan ERGE más comúnmente y se benefician más de un tratamiento antirreflujo como el TIF.

Chandan, et al. ${ }^{3}$ realizaron una revisión sistemática y metaanálisis comparando resultados y eventos adversos de la miotomía esofágica corta (SM) vs. standard (STM) en acalasia. Cinco estudios incluidos (450 pacientes); 205 SM y 245 STM, la longitud de la miotomía gástrica fue de 2-3.2 cm, el tipo de acalasia más común fue el tipo II, la estancia intrahospitalaria $(E I H)$ fue de 2.8 a 9.9 días y el seguimiento de 6 a 29.5

\section{Correspondencia:}


meses en promedio en ambos grupos. No se observó diferencia en el éxito clínico (OR: 1.29; IC 95\%: 0.572.91), síntomas de ERGE posquirúrgicos (OR: 0.87; IC 95\%: 0.44-1.74). La esofagitis fue relativamente menor en el grupo SM (OR: 0.50; IC 95\%: 0.24-1.03; $p=0.06$ ). La miotomía corta no es mejor que la clásica para el POEM en pacientes con acalasia.

Nullens, et al. ${ }^{4}$ estudiaron la prevalencia de eventos adversos (EA) tempranos y la capacidad de la endoscopia y esofagograma para su detección de forma retrospectiva y unicéntrica. Se incluyeron 358 pacientes. El esofagograma se realizó a las $24 \mathrm{~h}$ post-POEM en 162 pacientes los primeros 5 años, abandonando posteriormente esta práctica, se documentó fuga en el $1.85 \%$ de los casos. La endoscopia post-POEM se realizó en los primeros 14 pacientes a las $48 \mathrm{~h}$ y no hubo hallazgos relevantes, por lo cual se suspendió su uso posterior. Se realizaron 190 POEM adicionales sin esofagograma ni endoscopia de revisión. La prevalencia de EA tempranos fue de $19.5 \%$ (capnoperitoneo con necesidad de drenaje en $6.25 \%$; mucosotomías distales en $4.82 \%$ como los EA más relevantes). No hubo muertes O EA. EI POEM es un procedimiento seguro, el uso de esofagograma y endoscopia post-POEM no son necesarios a menos que exista una sospecha clínica de potencial EA.

Calderon, et al..$^{5}$ realizaron un estudio retrospectivo evaluando la eficacia y seguridad del POEM de repetición (redo-POEM) en pacientes con síntomas refractarios/recurrentes al POEM inicial. 15 pacientes incluidos, todos estos con falla al tratamiento (Eckardt $>6$ puntos, IRP $>15 \mathrm{mmHg}$ ). El abordaje fue posterior y el seguimiento de 6-52 meses. El 53.3\% tenían acalasia tipo II, $26.7 \%$ acalasia no especificada, $20 \%$ tipo I. Eckardt inicial $7.1 \pm 2.3$. Al comparar parámetros entre POEM inicial y redo-POEM se documentó un tiempo del procedimiento de 61.9 vs. 62.2 min ( $p=0.96$ ); miotomía de 6.9 vs. $6.1 \mathrm{~cm}(p=0.13)$. Éxito clínico en 10/15 redo-POEM $(67 \%)$, de los que el $57 \%$ tuvo mejora $<6$ meses y el $43 \%>6$ meses, y de estos últimos el $67 \%$ continuó con mejora hasta 31 meses posteriores al redo-POEM. La redo-POEM es una alternativa segura y efectiva en pacientes con acalasia recurrente/refractaria a POEM inicial.

Ghazaleh, et al. ${ }^{6}$ llevaron a cabo una revisión sistemática y metaanálisis comparando POEM y miotomía de Heller (MH) en acalasia. 19 estudios encontrados (1,799 pacientes). EI POEM demostró un mayor éxito clínico (OR: 1.65; 1.18- 2.32), menor estancia intrahospitalaria (OR: $-0.44 ;-0.87$ a -0.01 ), y menor tiempo quirúrgico (OR: -23.25; -37.86 a -8.44). Similares EA (OR: $0.76 ; 0.50-1.15)$, y mayor reflujo posterior (OR: 1.76; 1.03-3.0). Por lo cual el POEM podría considerarse el tratamiento de primera línea en acalasia esofágica considerando solo una mayor tasa de reflujo posterior.

\section{Miotomía pilórica endoscópica peroral (G-POEM)}

Shah, et al. ${ }^{7}$ evaluaron la realización de G-POEM de forma ambulatoria. Se incluyeron 25 pacientes, 9 egresados el mismo día y 16 ingresados $>24$ h para vigilancia, la estratificación fue basada en el índice de comorbilidades de Charlson, el cual fue claramente menor en el grupo de pacientes ambulatorios $(p<0.05)$. Éxito técnico de $100 \%$ y clínico de $81 \%$ al mes del G-POEM. Los EA se presentaron en el $12 \%$ de los casos, todos leves. Se concluye que el G-POEM ambulatorio es factible en pacientes seleccionados.

Welinsky, et al. ${ }^{8}$ reportó de forma retrospectiva y unicéntrica la experiencia del redo G-POEM. Documentó 3 pacientes, de los cuales 2 tuvieron un abordaje por curvatura mayor (GC) y uno por curvatura menor (LC), durante el primer G-POEM. No se reportaron EA y se mantuvo respuesta clínica por 3-17 meses. Se realizó el redo G-POEM en de 14 a 18 meses posterior al inicial por recurrencia clínica, el cual se realizó de forma contralateral al abordaje del G-POEM inicial; se documentaron 2 EA (1 hemorragia y 1 dehiscencia), los cuales se manejaron endoscópicamente. Se reportó una mejora del puntaje clínico en 2/3 de los pacientes a los 2 y 6 meses del redo G-POEM. Por lo cual se concluye que esta es una técnica de salvamento segura y potencialmente efectiva ante un G-POEM fallido inicial.

\section{Miotomía peroral endoscópica del Zenker (Z-POEM)}

Swei, et al. ${ }^{9}$ compararon septotomía flexible endoscópica (FES) contra Z-POEM. Diez pacientes sometidos a Z-POEM y 20 a FES, ambos con características basales similares. Tiempo de procedimiento de $45.6 \mathrm{mi}-$ nutos y 56.7 en Z-POEM y FES respectivamente ( $p=0.153$ ). El éxito técnico fue de $100 \%$ en ambos grupos, sin diferencia significativa en el puntaje de disfagia ( 0.2 en Z-POEM vs. 1.0 en FES; $p=0.257$ ), no hubo EA severos reportados. Por lo cual concluyen que tanto la FES como el Z-POEM presentan resultados 
similares en términos técnicos y clínicos para el tratamiento del Zenker.

\section{Miotomía peroral endoscópica cricofaríngea (C-POEM)}

$\mathrm{Wu}$, et al. ${ }^{10}$ evaluaron la factibilidad del C-POEM en el tratamiento de la disfagia orofaríngea por disfunción del esfínter esofágico superior (EES) en enfermedad de Parkinson. Se realizó una manometría con impedancia de alta resolución faríngea (HRPIM), un cuestionario de deglución de Sydney (SSQ) y de calidad de vida de la deglución (SwalQol) pre y post-C-POEM $1 \mathrm{~m}$. Ocho pacientes presentaron disfunción del EES y 6 incluidos, sin EA. Los puntajes de SSQ y SwalQol mejoraron en el 54 y $20 \%$ respectivamente. La HRPIM confirmó disminución del tono basal $(61.2$ a $8.7 \mathrm{mmHg})$, presión de relajación integrada $(12.6 \mathrm{a}-0.3 \mathrm{mmHg})$ y presión intrabolo hipofaríngea (28 a $13.7 \mathrm{mmHg}$ ). Por lo cual C-POEM es una alternativa potencialmente segura y efectiva en este grupo de pacientes.

\section{Financiamiento}

La presente investigación no ha recibido ayudas específicas provenientes de agencias del sector público, sector comercial o entidades sin ánimo de lucro.

\section{Conflicto de intereses}

Los autores declaran que no hay conflicto de intereses.

\section{Bibliografía}

1. Stavropoulos SN, Parsa N, Omrani L, Kollarus M, Peller H, Widmer JL, et al. ID: 3526903 Unlike heller myotomy (HM), per oral endoscopic myotomy (POEM) is associated with improvement in objective gastroesophageal reflux disease metrics on long term follow-up. Gastrointest Endosc [Internet]. 2021;93(6 Suppl):AB307. Disponible en: https://www. giejournal.org/article/S0016-5107(21)00872-5/fulltext

2. Kolb J, Nguyen P, Ji SS, Samarasena JB, Chang KJ. ID: 3522391 The risk of needing an antireflux procedure after per oral endoscopic myotomy and salvage with transoral incisionless fundoplication. Gastrointest Endosc [Internet]. 2021;93(6 Suppl):AB307. Disponible en: https://www. giejournal.org/article/S0016-5107(21)00873-7/abstract

3. Chandan S, Facciorusso A, Khan S, Ramai D, Mohan BP, Bilal M, et al. ID: 3521961 Short vs. standard esophageal myotomy in achalasia a systematic review and meta-analysis of comparative trials. Gastrointest Endosc [Internet]. 2021;93(6 Suppl):AB305-AB306. Disponible en: https://www.giejournal.org/article/S0016-5107(21)00870-1/fulltext

4. Nullens S, Nijhuis R, Oors J, Bastiaansen BA, Fockens P, Bredenoord A et al. ID: 3520717 Early adverse events following poem and the role of post-procedural imaging and endoscopy. Gastrointest Endosc [Internet]. 2021;93(6 Suppl):AB301-AB302. Disponible en: https://www.giejournal. org/article/S0016-5107(21)00863-4/abstract

5. Calderon LF,Veeramachaneni $H$, Samo $S$, Messallam AA, Shah R, Keilin S, et al. ID: 3523846 Long term outcomes of redo poems in patients with refractory or recurrent achalasia: a single center experience. Gastrointest Endosc [Internet]. 2021;93(6 Suppl):AB301-AB302. Disponible en: https://www.giejournal.org/article/S0016-5107(21)00855-5/fulltext

6. Ghazaleh Sami, Beran Azizullah, Pate Dipen, et al. Peroral endoscopic myotomy (POEM) vs. laparoscopic Heller myotomy (LHM) for esophageal achalasia a systematic review and meta-analysis. Presentado en cartel virtual durante la DDW; 2021, mayo 21-23, ePoster Su152.

7. Shah R, Calderon L, Li X, Alceguire K, Xie M, Messallam A, et al. ID 3522594 Safety and feasibility of same day discharge after per oral endoscopic pyloromyotomy in refractory gastroparesis: a pilot study. Gastrointest Endosc [Internet]. 2021;93(6 Suppl):AB9. Disponible en: https:// www.giejournal.org/article/S0016-5107(21)00329-1/fulltext

8. Welinsky S, Wynter J, Jodorkovsky D, Sethi A. ID: 3526796 Repeat gastric peri-oral endoscopic myotomy for treatment of gastroparesis: a case series. Gastrointest Endosc [Internet]. 2021;93(6 Suppl):AB311. Disponible en: https://www.giejournal.org/article/S0016-5107(21)00879-8/fulltext

9. Swei E, Wagh M. ID: 3525909 Comparison of Zenker's Per-oral Endoscopic Myotomy (Z-POEM) with traditional flexible endoscopic septotomy. Gastrointest Endosc [Internet]. 2021;93(6 Suppl):AB312-313. Disponible en: https://www.giejournal.org/article/S0016-5107(21)00882-8/abstract

10. Wu PI, SzczesniakM, Wong M, Omari T, Mok V, Cook IJ, et al. ID: 3522464 Cricopharyngeal Peroral Endoscopic Myotomy (C-POEM) for dysphagic Parkinson's disease patients with impaired cricopharyngeal relaxation. Gastrointest Endosc [Internet]. 2021;93(6 Suppl):AB302. Disponible en: https:// www.giejournal.org/article/S0016-5107(21)00864-6/abstract 